\title{
Periodic bouncing modes for two uniformly magnetized spheres. II. Scaling
}

Cite as: Chaos 30, 013131 (2020); https://doi.org/10.1063/1.5125925

Submitted: 27 August 2019 . Accepted: 30 December 2019 . Published Online: 21 January 2020

Boyd F. Edwards (D), Bo A. Johnson (D), and John M. Edwards (D)
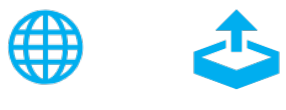

\section{ARTICLES YOU MAY BE INTERESTED IN}

Periodic bouncing modes for two uniformly magnetized spheres. I. Trajectories

Chaos: An Interdisciplinary Journal of Nonlinear Science 30, 013146 (2020); https:// doi.org/10.1063/1.5125924

Identifying characteristic time scales in power grid frequency fluctuations with DFA Chaos: An Interdisciplinary Journal of Nonlinear Science 30, 013130 (2020); https:// doi.org/10.1063/1.5123778

The maximum likelihood climate change for global warming under the influence of greenhouse effect and Lévy noise

Chaos: An Interdisciplinary Journal of Nonlinear Science 30, 013132 (2020); https:// doi.org/10.1063/1.5129003

\section{Scilight}




\title{
Periodic bouncing modes for two uniformly magnetized spheres. II. Scaling
}

\author{
Cite as: Chaos 30, 013131 (2020); doi: 10.1063/1.5125925 \\ Submitted: 27 August 2019 . Accepted: 30 December 2019 . \\ Published Online: 21 January 2020
}

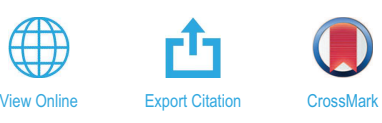

Boyd F. Edwards, ', a) (D) Bo A. Johnson, ? (D) and John M. Edwards ${ }^{2}$ (D)

\author{
AFFILIATIONS \\ ${ }^{1}$ Department of Physics, Utah State University, Logan, Utah 84322, USA \\ ${ }^{2}$ Department of Computer Science, Utah State University, Logan, Utah 84322, USA
}

a) Author to whom correspondence should be addressed: boyd.edwards@usu.edu

\begin{abstract}
A uniformly magnetized sphere moves without friction in a plane in response to the field of a second, identical, fixed sphere and makes elastic hard-sphere collisions with this sphere. Numerical simulations of the threshold energies and periods of periodic finite-amplitude nonlinear bouncing modes agree with small-amplitude closed-form mathematical results, which are used to identify scaling parameters that govern the entire amplitude range, including power-law scaling at large amplitudes. Scaling parameters are combinations of the bouncing number, the rocking number, the phase, and numerical factors. Discontinuities in the scaling functions are found when viewing the threshold energy and period as separate functions of the scaling parameters, for which large-amplitude scaling exponents are obtained from fits to the data. These discontinuities disappear when the threshold energy is viewed as a function of the threshold period, for which the large-amplitude scaling exponent is obtained analytically and for which scaling applies to both in-phase and out-of-phase modes.
\end{abstract}

Published under license by AIP Publishing. https://doi.org/10.1063/1.5125925

The purpose of this work is to investigate the scaling relationships between the threshold energy, the threshold period, the bouncing number, the rocking number, and the phase of 1497 periodic modes found previously for the motion of a uniformly magnetized sphere subject to the field of a second, identical, fixed sphere. This large dataset offers the opportunity to identify scaling relationships to high precision for this highly nonlinear problem. Such scaling relationships recall techniques used in studying phase transitions and fractals and invite the search for universal scaling laws that may also apply to other systems. This work is motivated by our interest in the properties of collections of small neodymium magnet spheres that are used to create beautiful magnetic sculptures and are used both in and out of the classroom to teach principles of mathematics, physics, chemistry, biology, and engineering.

\section{INTRODUCTION}

In Paper I, ${ }^{1}$ we use MagPhyx software to find 1497 periodic modes for a uniformly magnetized sphere moving without friction in response to the field of a second, identical, fixed sphere and making elastic hard-sphere collisions with this sphere. ${ }^{2}$ Three quantities $(m, n, p)$ characterize these modes: (1) the number $m$ of collisions during one period of the motion, called the bouncing number, (2) the number $n$ of angular oscillations during one period of the motion, called the rocking number, and (3) the initial phase of the angular oscillations, with $p=1$ for the in-phase motion with initial orbital and spin angular momenta of the same sign and with $p=2$ for the out-of-phase motion with initial orbital and spin angular momenta of opposite signs.

As discussed in Ref. 1, the basis for all of these modes is the finite-amplitude radial mode. In this mode, the magnetic moments of the two spheres remain in the same direction as the motion of the free sphere-there is no angular motion as the south pole of the free sphere bounces repeatedly against the north pole of the fixed sphere. As the energy of this mode increases, bifurcations to other modes $(m, n, p)$ occur at threshold energies $E_{m n p}$ and periods $T_{m n p}$. At these bifurcations, the angular motion is infinitesimal, with $p_{\phi}(0) \rightarrow 0$ and $p_{\theta}(0) \rightarrow 0$. As the energy increases beyond this threshold for a particular mode $(m, n, p)$, the amplitude of the angular motion increases. Thus, the threshold energy $E_{m n p}$ and period $T_{m n p}$ correspond to the finite-amplitude radial motion and infinitesimal-amplitude angular motion.

The purpose of this paper is to investigate how $E_{m n p}$ and $T_{m n p}$ depend on $m, n$, and $p$ for the motion with small, intermediate, and 
large amplitudes. Values of $E_{m n p}$ range between $-1 / 3$ and 0 , with $E=-1 / 3$ giving the stable equilibrium state with the south pole of the free magnet in permanent contact with the north pole of the fixed magnet and with $E=0$ giving the upper energy limit for bound states. Energies near $E=-1 / 3$ give small-amplitude motions for which the free sphere remains in the vicinity of its stable equilibrium position, while energies near $E=0$ give large-amplitude nonlinear motions for which the free sphere wanders far from this position.

In this paper, we test our data against mathematical predictions of $E_{m n p}$ and $T_{m n p}$ for small amplitudes and use scaling parameters identified by these predictions to propose and test power-law scaling for large amplitudes. The use of scaling parameters and the investigation of large-amplitude power-law scaling draws upon techniques used to study phase transitions and random fractals.

In this study, we take advantage of our recent proof that simple dipolar interactions exactly describe the magnetic interactions between uniformly magnetized spheres ${ }^{4}$ and build upon our subsequent studies of dynamical interactions between spheres that remain in contact, both with and without friction. ${ }^{5,6}$

Our 1497 modes exhibit a wide variety of behaviors, including simple modes with small $m$ and $n$ and complicated modes with large $m$ and $n$. Mode $(157,580,2)$ is the most complicated that we discovered. With $E_{m n p}=-0.003999$ and $T_{m n p}=11298$, this mode requires about 16000 Runge-Kutta time steps to integrate. Tracking changes in the energy provides a valuable test of the reliability of the integration of our conservative system of equations. For the longperiod $(157,580,2)$ mode, the energy variations are within $10^{-10}$. Energy variations are even smaller for simpler modes with shorter periods.

Collections of small neodymium magnet spheres are used to create beautiful magnetic sculptures; ${ }^{7}$ to teach principles of mathematics, physics, chemistry, biology, and engineering, ${ }^{8}$ and to investigate the properties of chains and rings. ${ }^{9-17}$

\section{FINITE-AMPLITUDE RADIAL MODE}

The basis for scaling for threshold states is the finite-amplitude radial mode. In this section, we relate the period $T$ of this mode to its energy $E$ and infer a relationship between the threshold energy $E_{m n p}$ and period $T_{m n p}$ of mode $(m, n, p)$.

Setting $\theta=\phi=p_{\theta}=p_{\phi}=0$ in Eqs. (11a) and (11b) in Ref. 1 gives the total energy of the finite-amplitude radial mode,

$$
E=\frac{\dot{r}^{2}}{2}-\frac{1}{3 r^{3}}
$$

As we did for our small-amplitude investigations (Ref. 1, Sec. III A), we employ the initial conditions $r(0)=1$ and $\dot{r}(0)=v_{0}$, placing the free sphere in instantaneous initial contact with the fixed sphere. We integrate until the time $t=T / 2$ is half the period $T$, at which point the free sphere is located at its maximum radius $r(T / 2)=a$, and its speed is $\dot{r}(T / 2)=0$. Evaluating the (conserved) total energy at $t=T / 2$ gives a relationship between $E$ and $a$,

$$
E=-\frac{1}{3 a^{3}}
$$

where bound periodic states satisfy $-1 / 3<E<0$ and $1<a<\infty$.
Combining Eqs. (1) and (2), rearranging, and integrating over half a period gives

$$
\int_{1}^{a} \frac{d r}{\sqrt{r^{-3}-a^{-3}}}=\sqrt{\frac{2}{3}} \int_{0}^{T / 2} d t
$$

Conveniently substituting $\rho=r / a$ to rewrite the integral on the left side yields the period $T$ as a function of the maximum radius $a$,

$$
T=\sqrt{6} a^{5 / 2} f(a)
$$

where the integral

$$
f(a)=\int_{1 / a}^{1} \frac{d \rho}{\sqrt{\rho^{-3}-1}}
$$

cannot generally be expressed in terms of elementary functions. We use Maple software to evaluate the integral, which has the limiting values $f(1)=0$ and

$$
f(\infty)=\int_{0}^{1} \frac{d \rho}{\sqrt{\rho^{-3}-1}}=0.7468 .
$$

At its threshold energy $E_{m n p}$, mode $(m, n, p)$ executes the same motion as the finite-amplitude radial mode of the same energy $E$ but executes it $m$ times, apart from its $n$ infinitesimal-amplitude angular oscillations. Consequently, if $E_{m n p}=E$, then $T_{m n p}=m T$, and Eqs. (2) and (4) imply

$$
\begin{gathered}
E_{m n p}=-\frac{1}{3 a^{3}}, \\
T_{m n p}=m \sqrt{6} a^{5 / 2} f(a) .
\end{gathered}
$$

These equations relate the threshold energy and period of mode $(m, n, p)$ through the maximum radius $a$.

\section{SMALL-AMPLITUDE SCALING}

For small-amplitude radial motion, $a=1+\delta$, where $\delta \ll 1$. Working to the lowest order in $\delta$, Eqs. (7) and (8) reduce to

$$
\begin{aligned}
& E_{m n p}=-\frac{1}{3}+\delta, \\
& T_{m n p}=2 m \sqrt{2 \delta},
\end{aligned}
$$

where we have integrated Eq. (5) by substituting $\rho=1-\zeta$ with $\zeta \ll 1$, yielding

$$
f(\delta)=\int_{0}^{\delta} \frac{d \zeta}{\sqrt{3 \zeta}}=2 \sqrt{\frac{\delta}{3}} .
$$

During one period of the motion for mode $(m, n, p)$, the free sphere executes $n$ angular oscillations. For small-amplitude radial and angular oscillations, the period of each angular oscillation is given by the period $T_{p}$ of the small-amplitude sliding motion for 
which the two spheres remain in contact at all times, with ${ }^{6}$

$$
T_{1}=2 \pi \sqrt{\frac{6}{13-\sqrt{139}}} \approx 14,
$$

giving the period of in-phase oscillations and

$$
T_{2}=2 \pi \sqrt{\frac{6}{13+\sqrt{139}}} \approx 3.1,
$$

giving the period of out-of-phase oscillations. Accordingly setting $T_{m n p}=n T_{p}$ allows us to rewrite Eqs. (9) and (10) as ${ }^{1}$

$$
\begin{gathered}
E_{m n p}=-\frac{1}{3}+\frac{n^{2} T_{p}^{2}}{8 m^{2}}, \\
T_{m n p}=n T_{p},
\end{gathered}
$$

valid for $m \gg n$. The condition $m \gg n$ means that small-amplitude periodic states have large bouncing numbers $m$ compared with their rocking numbers $n$. In these states, the bouncing frequency is high and the free sphere remains close to the fixed sphere.

Figure 1 tests these predictions against our finite-amplitude simulations for rocking number $n=1$ and bouncing numbers $m=1,2,3, \ldots, 100$ for both the in-phase motion $(p=1)$ and outof-phase motion $(p=2)$. This test shows excellent agreement for $m \gg n$ and helps us to validate our simulation code for $n=1$.

Figure 2 shows $E_{m n p}$ vs $T_{m n p} / T_{2}$ for various bouncing numbers $m$ and rocking numbers $n$ for the in-phase ( $p=1$, open circles) and the out-of-phase ( $p=2$, filled circles) motions. Solid constant- $m$ traces are given by Eqs. (7) and (8), with $f(a)$ evaluated numerically; values of $m$ for each trace are given along the right side of the plot. The leftmost data point on each solid trace marks an out-of-phase mode with $n=1$. To its right along each solid trace are out-of-phase modes with increasing values of $n$, interrupted occasionally by inphase modes. Also included are predicted results for $m=997$ given by Eqs. (14) and (15). Our data for increasing $m$ clearly approach the predicted results for $m=997$. This approach helps us to validate our simulation code for $n \geq 1$, as does the agreement between the solid traces and the data points.

For small-amplitude out-of-phase modes with $p=2$, Eq. (15) implies that the scaled period $T_{m n 2} / T_{2}=n$ is just the associated rocking number $n$, which can therefore be read directly from the horizontal axis labels. For in-phase modes with $p=1$, the quantity $T_{m n 1} / T_{2}=n T_{1} / T_{2}$ is the product of the rocking number $n$ and the ratio $T_{1} / T_{2}=4.53$. Rocking numbers $n$ for in-phase modes (open circles) are shown in parentheses above the horizontal axis. In this way, families of modes with the same value of $n$ can be identified by where they meet the horizontal axis.

As seen in Fig. 2, $E_{m n p}$ is always negative, approaching zero for small $m$ and decreasing with increasing $m$, approaching $E_{m n p} \rightarrow-1 / 3$ as $m \rightarrow \infty$ for both in-phase and out-of-phase modes [Eq. (14)]. For fixed $m, E_{m n p}$ increases with $T_{m n p}$.

Dashed horizontal lines in Fig. 2 point from duplicate modes to the associated irreducible modes of the same energy. ${ }^{1}$ For example, the dashed horizontal line at $E=-0.065$ starts the irreducible mode $(1,1,2)$ and passes through the duplicate modes $(2,2,2),(3,3,2)$, etc., of the same energy. The periods of these duplicate modes

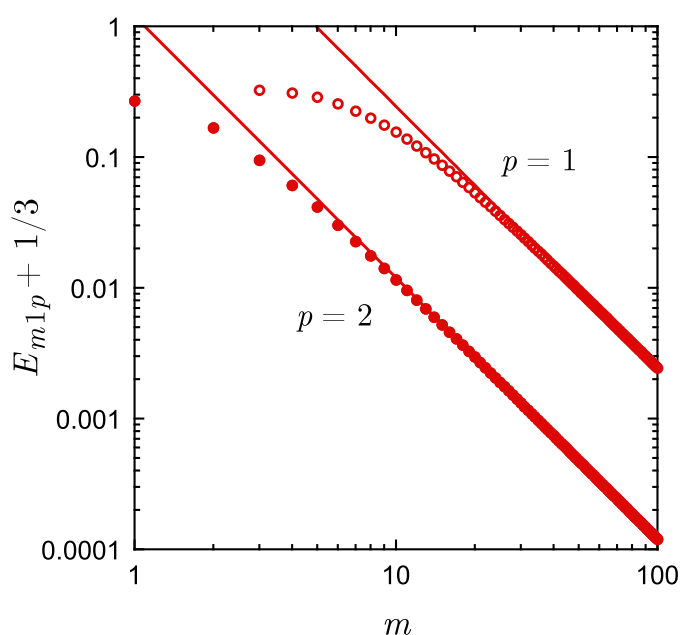

(a)

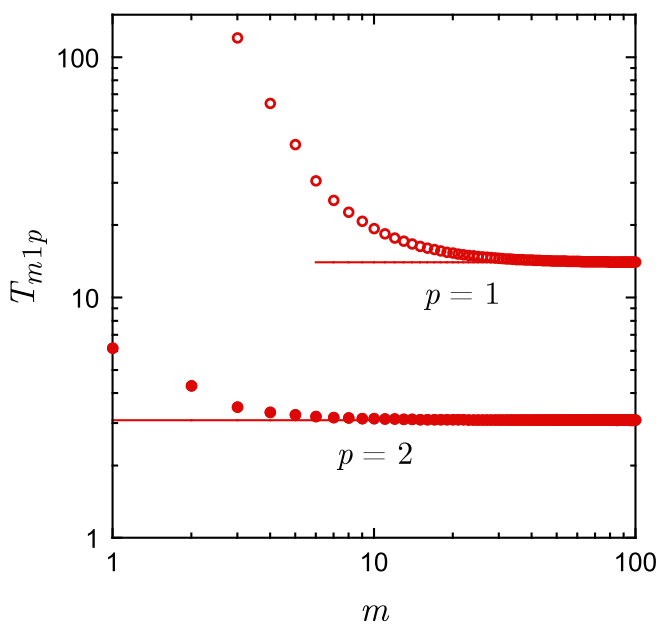

(b)

FIG. 1. Threshold energy $E_{m n p}$ (a) and period $T_{m n p}$ (b) vs bouncing number $m$ for periodic states with rocking number $n=1$, for spin and orbital motions that are in phase $(p=1)$ and out of phase $(p=2)$. Data points are results from our finite-amplitude simulations, and solid traces are given by Eqs. (14) and (15), valid for $m \gg n$.

obey $T_{m, m, 2}=m T_{1,1,2}$ with $m>1$ being integer multiples of the period $T_{1,1,2}$ of the irreducible mode. This is because duplicate mode $(m, m, 2)$ simply replicates, $m$ times, the motion of the irreducible mode $(1,1,2)$.

\section{LARGE-AMPLITUDE SCALING}

In this section, we seek to identify scaling parameters that apply to the entire range of amplitudes and to identify large-amplitude scaling behavior in the data. For this purpose, it is helpful to rewrite 


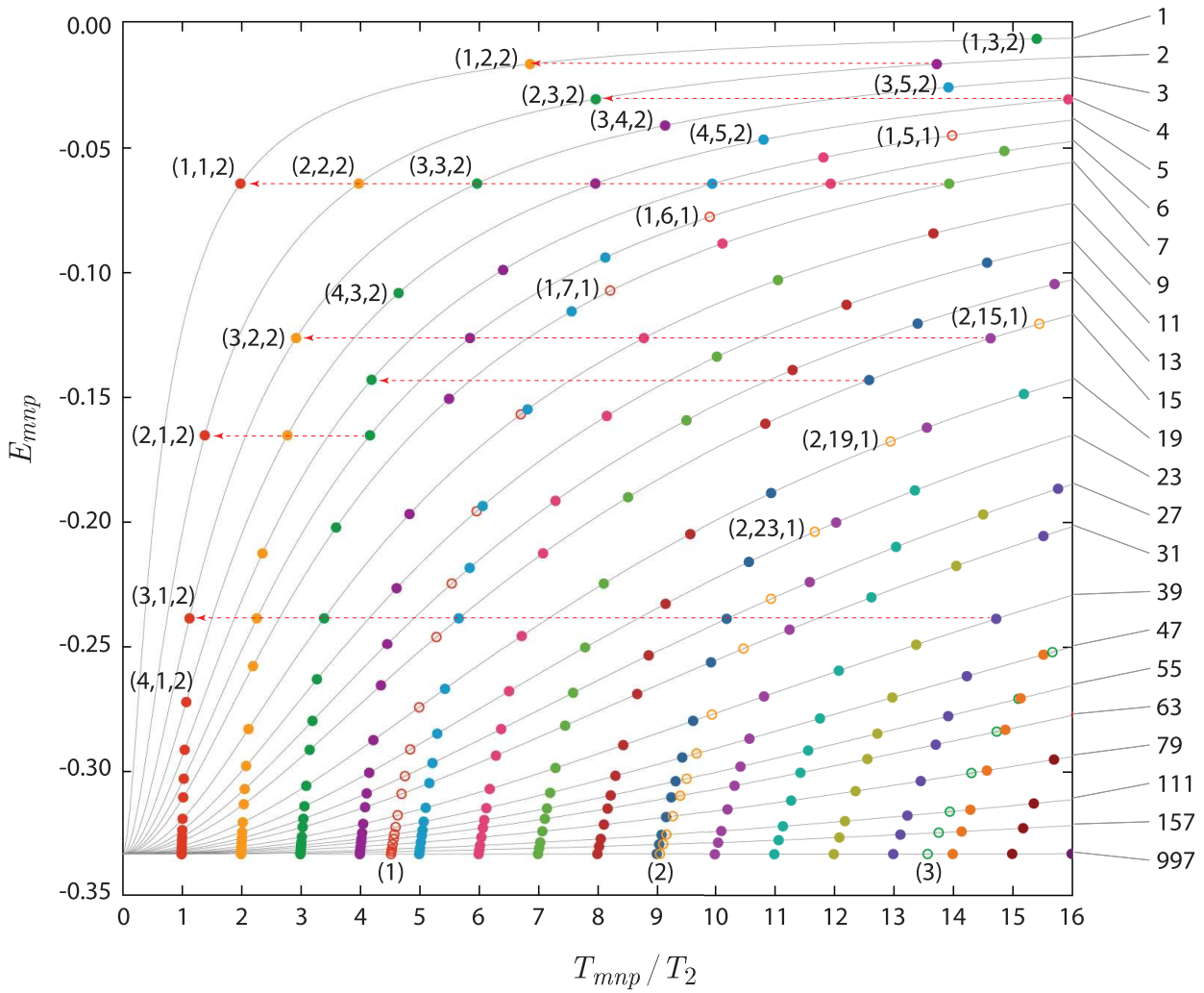

FIG. 2. Threshold energy $E_{m n p}$ vs threshold period $T_{m n p}$ scaled by the period $T_{2}$ of the small-amplitude out-of-phase sliding mode [Eq. (13)]. Solid traces are solutions of Eqs. (7) and (8) and are labeled by their bouncing numbers $m$ (numerical labels along the right side). Horizontal axis labels give rocking numbers $n$ for out-of-phase modes $(p=2$, filled circles) for $m \gg n$, and parenthesized integers give the rocking numbers $n$ for in-phase modes $(p=1$, open circles) in this limit. Data for $m=997$ are given by Eqs. (14) and (15), valid for small amplitudes and $m \gg n$. Data for all other values of $m$ are from our finite-amplitude simulations. Selected mode descriptors $(m, n, p)$ are shown to the left of the corresponding data points. Dashed horizontal lines point from duplicate modes to the associated irreducible modes of the same energy.

Eqs. (14) and (15) as

$$
\begin{gathered}
\tilde{E}_{m n p}=-\frac{1}{3}(1-\eta), \\
\tilde{T}_{m n p}=n T_{p},
\end{gathered}
$$

where

$$
\eta=\frac{3 n^{2} T_{p}^{2}}{8 m^{2}}
$$

is a scaling parameter that includes the bouncing number $m$, the rocking number $n$, and the period $T_{p}$ of the small-amplitude angular motion given by Eq. (12) for $p=1$ and by Eq. (13) for $p=2$. Equations (16) and (17) are valid for small amplitudes $\eta \ll 1$.

To investigate the scaling behavior of $E_{m n p}$ and $T_{m n p}$, we hypothesize that the variable $\eta$ governs the behavior of $E_{m n p}$ and $T_{m n p}$ not just for small amplitudes but over the entire range of amplitudes. For $E_{m n p}$, we propose the scaling form

$$
\tilde{E}_{m n p}(\eta)=-\frac{1}{3\left(1+\eta+\alpha_{p} \eta^{\beta_{p}}\right)},
$$

with fit constants satisfying $\alpha_{p}>0$ and $\beta_{p}>1$. This form reduces to Eq. (16) for $\eta \rightarrow 0$ and gives the power-law scaling relationship

$$
\tilde{E}_{m n p}=-\frac{1}{3 \alpha_{p}} \eta^{-\beta_{p}}
$$

for $\eta \rightarrow \infty$.
For $\tilde{T}_{m n p}$, we insert Eq. (19) into Eqs. (7) and (8)

$$
\tilde{T}_{m n p}(\eta)=m \sqrt{6} a^{5 / 2} f(a)
$$

where

$$
a=\left(-3 \tilde{E}_{m n p}\right)^{-1 / 3} .
$$

Equations (9) and (10) ensure that Eq. (21) reduces to Eq. (17) as $\eta \rightarrow 0$.

For $\eta \rightarrow \infty$, Eqs. (20)-(22) give power laws

$$
\begin{gathered}
a=\alpha_{p}^{1 / 3} \eta^{\beta_{p} / 3}, \\
\tilde{T}_{m n p}(\eta)=\sqrt{6} m f(\infty) \alpha_{p}^{5 / 6} \eta^{5 \beta_{p} / 6} .
\end{gathered}
$$

Figures 3 and 4 show fits of our simulation data to Eqs. (19) and (21), with values of the fit constants given in Table I. These fits are accomplished by minimizing

$$
\chi^{2}=\Sigma_{m n} \frac{\left(E_{m n p}-\tilde{E}_{m n p}\right)^{2}}{\left|E_{m n p}\right|^{3}},
$$

for $p=1$ and $p=2$, with uncertainties in the fit constants determined by varying each constant from its optimal value until $\chi^{2}$ increases by a factor of two. Figures 5 and 6 compare our simulation data to the predictions of Eq. (21).

Using $\left|E_{m n p}\right|^{2}$ as the denominator in Eq. (25) would assume that the uncertainties in $E_{m n p}$ are proportional to the quantities 


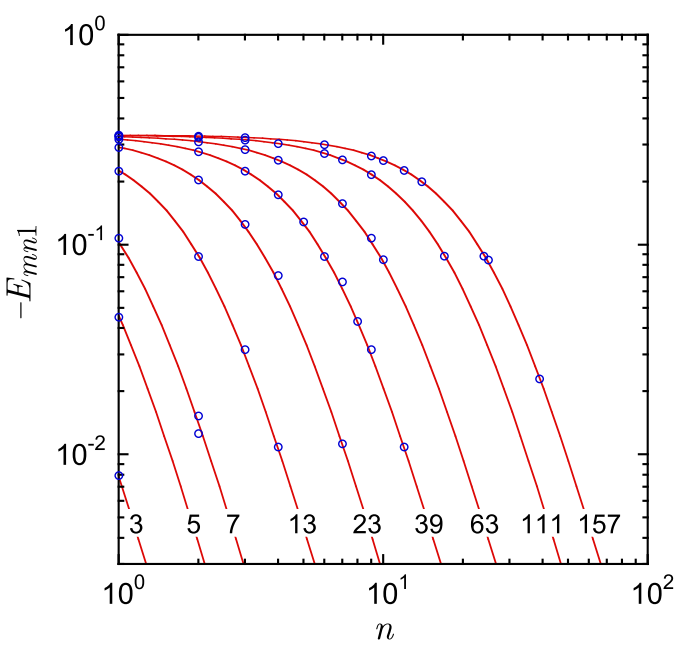

(a)

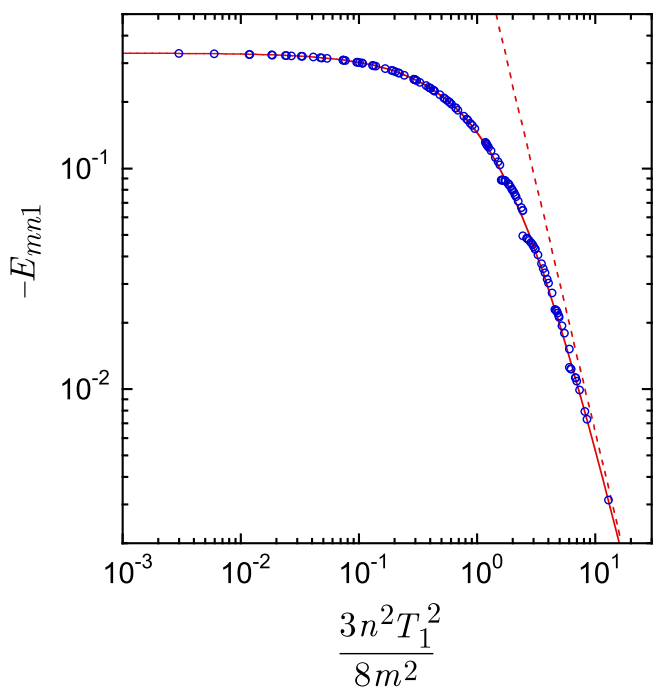

(b)

FIG. 3. (a) In-phase threshold energies $E_{m n 1}$ vs rocking number $n$, with simulation data shown as open circles and with Eq. (19) giving solid traces for constant bouncing numbers $m$, labeled as integers in the plot interior. (b) In-phase threshold energies $E_{m n 1}$ vs scaling parameter $\eta=3 n^{2} T_{1}^{2} / 8 m^{2}$, with simulation data for all values of $m$ shown as open circles, with Eq. (19) giving the solid trace, and with Eq. (20) giving the dashed trace.

themselves and would weight all data equally in the fits. Instead, to investigate large-amplitude scaling, we use $\left|E_{m n p}\right|^{3}$ as the denominator in order to weight the large-amplitude data more heavily in the fits, given that $E_{m n p} \rightarrow 0$ as $\eta \rightarrow \infty$. With nonstandard weights chosen to emphasize the large-amplitude data, Eq. (25) differs from the standard $\chi^{2}$ function, and the standard procedures for using the value of $\chi^{2}$ to test for goodness of fit do not apply.

The fits are made to all of our data, but only data for selected values of $m$ are shown in Figs. 3(a), 4(a), 5(a), and 6(a) for clarity.

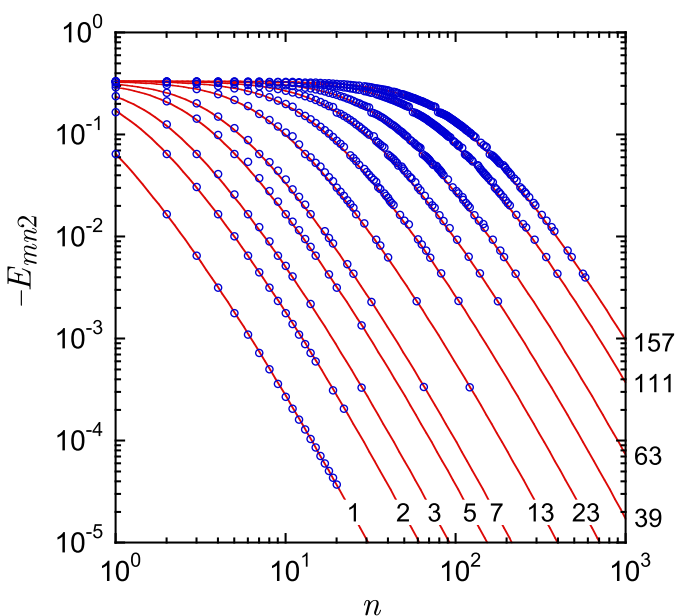

(a)

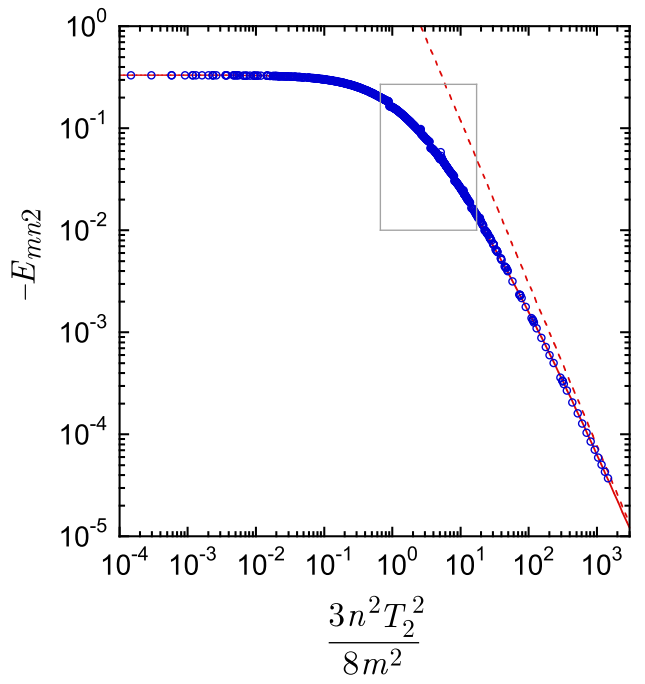

(b)

FIG. 4. (a) Out-of-phase threshold energies $E_{m n 2}$ vs rocking number $n$, with simulation data shown as open circles and with Eq. (19) giving solid traces for constant bouncing numbers $m$, labeled as integers in the plot interior. (b) Out-of-phase threshold energies $E_{m n 2}$ vs scaling parameter $\eta=3 n^{2} T_{2}^{2} / 8 m^{2}$, with simulation data for all values of $m$ shown as open circles, with Eq. (19) giving the solid trace, and with Eq. (20) giving the dashed trace. Figure 7 shows a close-up of the data within the gray rectangle.

TABLE I. Large-amplitude scaling constants resulting from fits of simulation data to Eq. (19).

\begin{tabular}{ccc}
\hline \hline$p$ & $\alpha_{p}$ & $\beta_{p}$ \\
\hline 1 & $0.29 \pm 0.03$ & $2.25 \pm 0.04$ \\
2 & $0.072 \pm 0.003$ & $1.592 \pm 0.005$ \\
\hline \hline
\end{tabular}




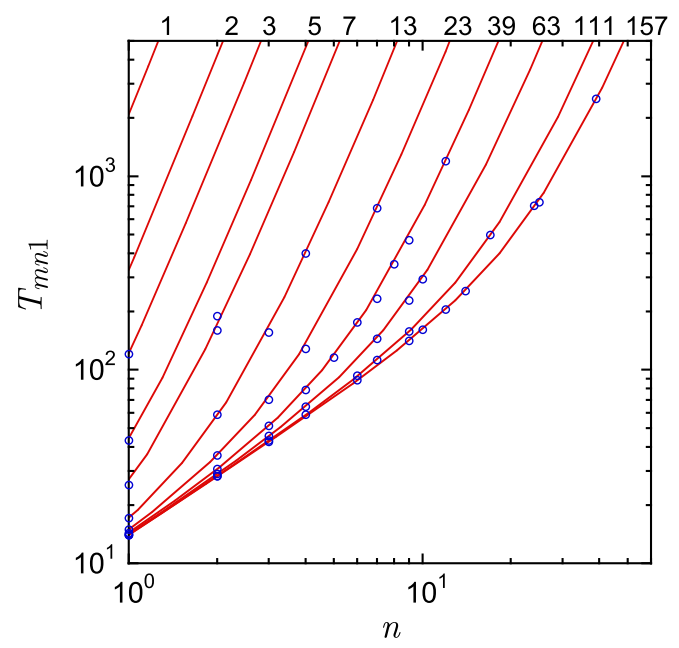

(a)

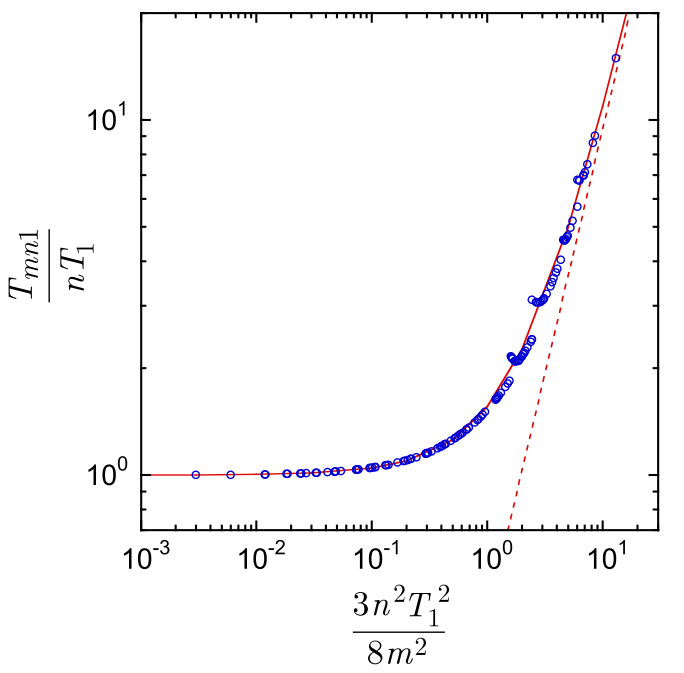

(b)

FIG. 5. (a) In-phase threshold periods $T_{m n 1}$ vs rocking number $n$, with simulation data shown as open circles and with Eq. (21) giving solid traces for constant bouncing numbers $m$, labeled as integers in the plot interior. (b) In-phase threshold periods $T_{m n 1}$ vs scaling parameter $\eta=3 n^{2} T_{1}^{2} / 8 m^{2}$, with simulation data for all values of $m$ shown as open circles, with Eq. (21) giving the solid trace, and with Eq. (24) giving the dashed trace.

A single equation, Eq. (19), with $p=1$ and just two fit parameters, $\alpha_{1}$ and $\beta_{1}$, describes all of the data in Fig. 3(a). A similar situation holds for Fig. 4(a) with Eq. (19), $p=2, \alpha_{2}$, and $\beta_{2}$. These fits provide evidence that $\eta$ serves as the pertinent scaling parameter for the data at all amplitudes and that $E_{m n p}$ and $T_{m n p}$ scale as powers of $\eta$ in the large-amplitude limit.

Figures 3(b), 4(b), 5(b), and 6(b), which include all of our data, supply further evidence that $\eta$ serves as the scaling parameter. These plots use $\eta=3 n^{2} T_{p}^{2} / 8 m^{2}$ as the horizontal coordinate and use $E_{m n p}$

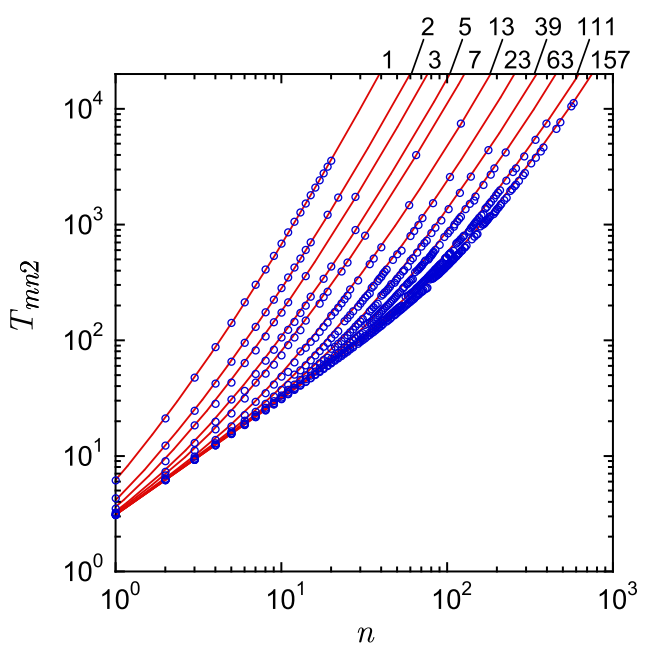

(a)

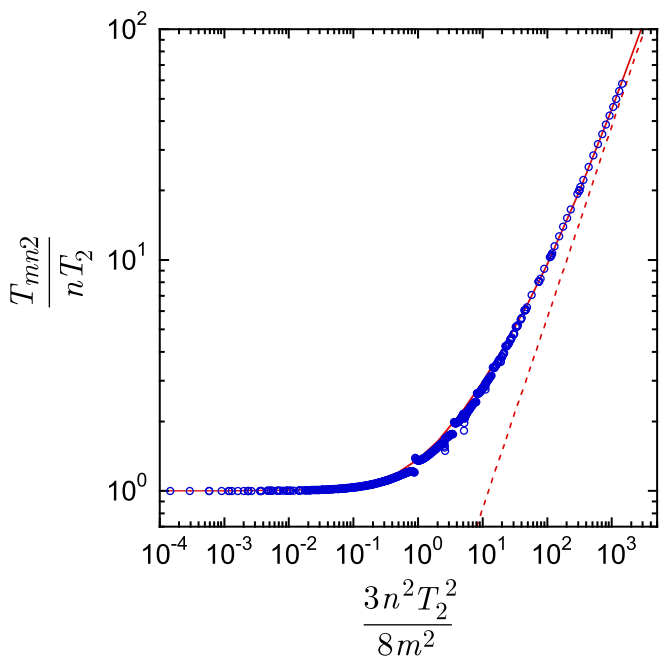

(b)

FIG. 6. (a) Out-of-phase threshold periods $T_{m n 2}$ vs rocking number $n$, with simulation data shown as open circles and with Eq. (21) giving solid traces for constant bouncing numbers $m$, labeled as integers in the plot interior. (b) In-phase threshold periods $T_{m n 2}$ vs scaling parameter $\eta=3 n^{2} T_{2}^{2} / 8 m^{2}$, with simulation data for all values of $m$ shown as open circles, with Eq. (21) giving the solid trace, and with Eq. (24) giving the dashed trace.

and $T_{m n p} / n T_{p}$ as the vertical coordinates in order to collapse all of the data onto a single trace, consistent with Eqs. (19) and (21).

Discontinuities appear in these plots at intermediate amplitudes and are remarkably honored by all of the data, which collapses down to a single trace in each case. These discontinuities, which are missed by the continuous scaling functions given by Eqs. (19) and (21), do not dismiss $\eta$ as the scaling parameter for the data but show only that these equations capture only the general behavior of the data and not its details. What matters in determining whether $\eta$ 


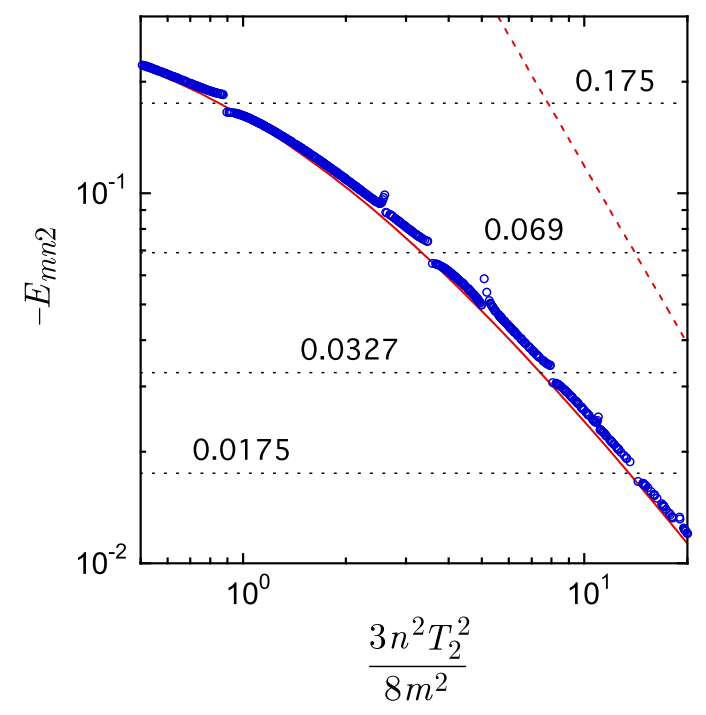

FIG. 7. Close-up of the data within the gray rectangle of Fig. 4(b), with discontinuities located at the intersections of the solid trace and the horizontal dotted lines, with decimal numbers giving the associated energy values.

serves as the scaling parameter is whether all of the data collapses to a single curve, albeit a discontinuous one, which it does.

Discontinuities can be seen in Figs. 3(b), 5(b), and 6(b) but are difficult to see in Fig. 4(b). To show that this figure also has discontinuities, we plot a close-up of the data in the gray rectangle to create Fig. 7, which clearly shows discontinuities.

In Fig. 3(a), the $m=7$ trace passes between two data points for $n=2$, which corresponds to the anomalous mode $(7,2,1)$, which describes two distinct families of states, each with its own threshold energy $E_{m n p}$, period $T_{m n p}$, and motion.

\section{UNIVERSAL SCALING}

We now consider the threshold energy as a function of the threshold period. To identify a convenient scaling parameter, we again appeal to the small-amplitude equations [Eqs. (14) and (15)], this time rewriting them as

$$
E_{m n p}=-\frac{1}{3}(1-\xi),
$$

where the combined parameter

$$
\xi=\frac{3 T_{m n p}^{2}}{8 m^{2}}
$$

involves only the threshold period $T_{m n p}$ and the bouncing number $m$. Equation (26) gives the relationship between the energy and the period for small amplitudes $\xi \rightarrow 0$.

The bouncing numbers in Eqs. (8) and (27) cancel after substituting Eq. (8) into (27), giving

$$
\xi=\frac{9}{4} a^{5} f^{2}(a),
$$

where Eq. (7) gives

$$
a=\left(-3 E_{m n p}\right)^{-1 / 3} \text {. }
$$

Equations (28) and (29) show that $E_{m n p}$ is a function of $\xi$ only and show that $\xi$ is the appropriate scaling parameter for $E_{m n p}$ when viewed as a function of $T_{m n p}$.

Equations (28) and (29) give the large-amplitude $(a \rightarrow \infty$, $\xi \rightarrow \infty)$ limit,

$$
E_{m n p}=-k \xi^{-3 / 5}
$$

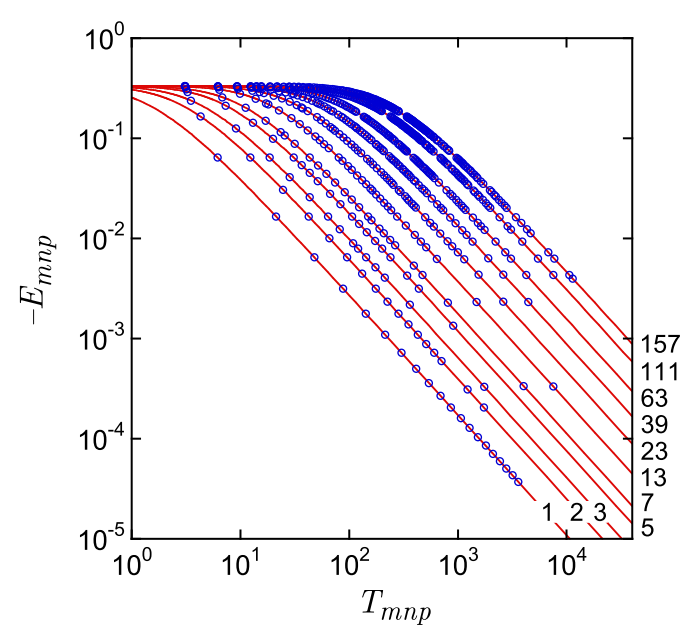

(a)

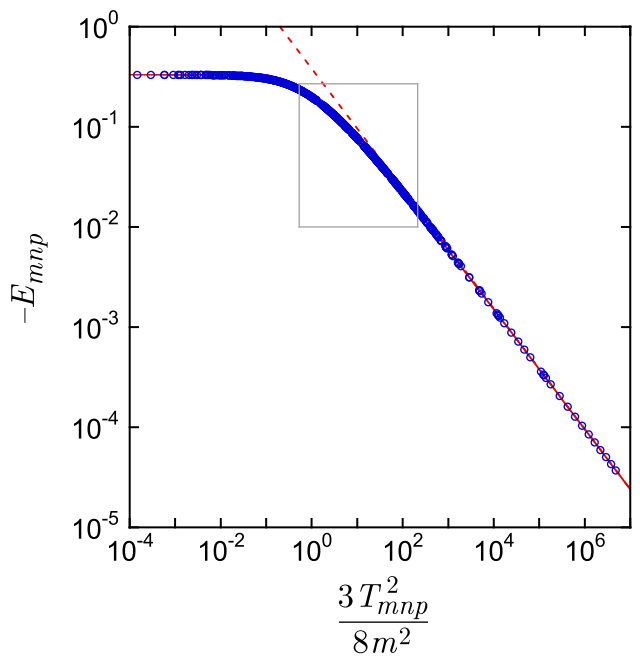

(b)

FIG. 8. (a) Threshold energies $E_{\text {mnp }}$ for both in-phase and out-of-phase modes vs threshold period $T_{m n p}$, with data shown as open circles and with solid traces for constant $m$ (integer labels) given by inserting Eq. (29) into Eq. (8). (b) Threshold energies $E_{m n p}$ vs scaling parameter $\xi=3 T_{m n p}^{2} / 8 m^{2}$, with simulation data for all values of $m$ shown as open circles and with Eqs. (8) and (29) giving the solid trace. Shown also is the large-amplitude scaling law given by Eq. (30) (dashed trace). Figure 9 shows a close-up of the data within the gray rectangle. 
where

$$
k=\frac{1}{3}\left[\frac{9 f^{2}(\infty)}{4}\right]^{3 / 5}=0.3820 .
$$

Evidently, $E_{m n p}$ obeys power-law scaling in $\xi$ for large amplitudes, with scaling exponent of $3 / 5$.

Figure 8 compares our simulation data for $E_{m n p}$ vs $T_{m n p}$ with the prediction of Eqs. (8) and (29), with only selected values of $m$ shown in Fig. 8(a) for clarity. Figure 8(b) shows all of our data (1497 modes in all). The scaling shown in Fig. 8 is stronger than the scaling shown in Figs. 3-6 because Fig. 8 includes both the in-phase and out-of-phase data, which are treated separately in Figs. 3-6. The agreement between the data and the predictions supplies evidence that the threshold energy $E_{m n p}$, when viewed as a function of the threshold period $T_{m n p}$, depends only on the parameter $\xi$ and that $E_{m n p}$ scales as a power of $\xi^{-3 / 5}$ for large amplitudes. This agreement also validates our Runge-Kutta integration of the equations of motion.

Figure 8(b) shows $E_{m n p}$ as a function of the scaling parameter $\xi$. The collapse of all of the data onto a single curve provides further evidence that $\xi$ is the appropriate scaling parameter. No discontinuities appear in this plot. Nor do any discontinuities appear in Fig. 9, a close-up view of the data within the gray rectangle of Fig. 8(b), which covers the same energies as Fig. 7. Although gaps in Fig. 9 coincide with discontinuities in Fig. 7, the data in Fig. 9 follow a smooth path with no discontinuities. Evidently, the energy discontinuities of Figs. 3 and 4 conspire with the period discontinuities of Figs. 5 and 6 to produce a smooth path when viewing the threshold energy as a function of the threshold period.

In Fig. 8(b), $-E_{m n p}$ decreases with increasing $T_{m n p}$ at constant $m$, meaning that $E_{m n p}$ increases with increasing $T_{m n p}$. Periods $T_{m n p}$ are small for the small-amplitude motion with energies $E_{m n p} \rightarrow-1 / 3$ and increase without bound as $E_{m n p} \rightarrow 0$ from below.

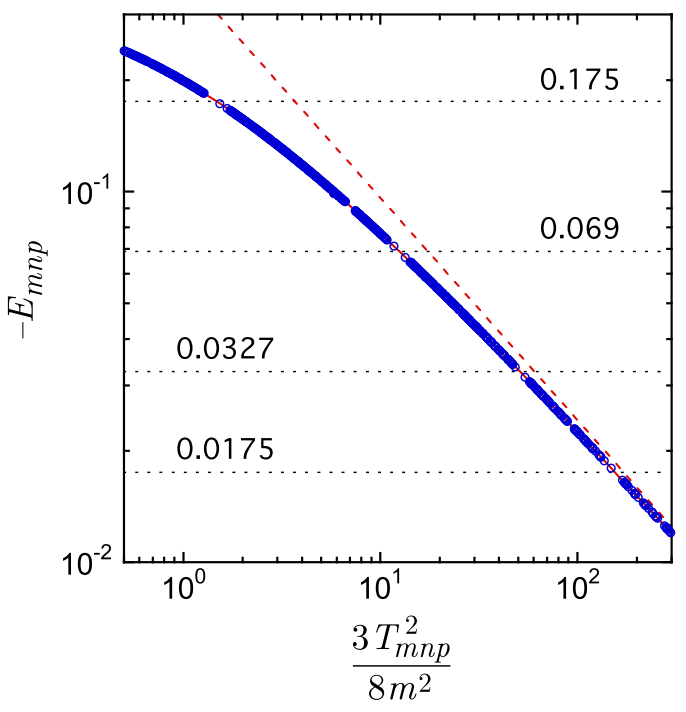

FIG. 9. Close-up of the data within the gray rectangle of Fig. $8(\mathrm{~b})$. Included are dotted horizontal lines at the energies of the discontinuities of Fig. 7.

\section{CONCLUSIONS}

At constant bouncing number $m$, the threshold period $T_{m n p}$ increases monotonically with increasing threshold energy $E_{m n p}$ and satisfies a simple scaling relationship governed by the scaling parameter $\xi=3 T_{m n p}^{2} / 8 m^{2}$. An entire dataset of 1497 periodic modes obeys this relationship, with a wide variety of bouncing numbers $m$, rocking numbers $n$, phases $p$, and trajectories. The collapse of all 1497 data points onto a single, smooth curve in Fig. 8(b) provides evidence of this relationship and seems remarkable given that each of these data points is the result of a numerical integration of the equations of motion with up to 16000 Runge-Kutta time steps.

This threshold energy-period scaling relationship originates from the energy-period relationship for the finite-amplitude radial bouncing mode, from which all periodic modes $(m, n, p)$ bifurcate. The former scaling relationship applies only to threshold energies $E_{m n p}$, that is, the minimum energy for mode $(m, n, p)$, and the associated period $T_{m n p}$. As the energy $E$ increases beyond this minimum for a particular mode, the period $T$ increases for some modes [such as $(2,1,2),(2,3,2)$, and $(3,1,2)]$ and decreases for others [such as $(1,1,2),(1,2,2),(2,5,2)]$.

Realizing intricate long-period bouncing modes experimentally seems challenging because of energy losses between and during collisions, but it may be possible to realize some simpler shortperiod modes experimentally.

Large hematite magnet spheres with diameters of the order of $2 \mathrm{~cm}$ might be better suited for such realizations than small neodymium magnets of diameter $0.5 \mathrm{~mm}$. A pair of such hematite magnets can collide repeatedly before settling into the stable equilibrium state, indicating that losses during collisions might be smaller for these magnets. Commercially, these magnets are called "rattlesnake eggs" because of the buzzing sound that they make as they collide repeatedly.

Attempting to observe bouncing modes under earth's gravity presents challenges. To confine the motion to a plane and to avoid gravitational deflections, one might carry out experiments on a horizontal surface, with one magnet affixed to the surface and the other free to slide along the surface. The associated kinetic friction acting on the free sphere would dissipate energy, violating the assumption of energy conservation. This friction would also exert a torque on the free sphere that would tend to rotate its magnetic moment out of the plane of motion, violating the assumption that both magnetic moments remain in the plane of motion.

An alternative is to carry out experiments in a drop tower or in orbit. For such experiments to replicate the calculations, one of the magnets would need to be held fixed by connecting it to a massive object using a rigid arm, for example. Such experiments would effectively eliminate friction between collisions but would still be subject to frictional losses during collisions. Such an arrangement could be used to investigate the $2 \mathrm{D}$ bouncing modes studied herein or $3 \mathrm{D}$ bouncing modes with three additional degrees of freedom (one translational and two rotational).

\section{REFERENCES}

${ }^{1}$ B. F. Edwards, B. A. Johnson, and J. M. Edwards, "Periodic bouncing modes for two uniformly magnetized spheres. I. Trajectories,” Chaos 30, 013146 (2020). 
${ }^{2}$ J. Edwards, "MagPhyx visualization software" (2019), see http://edwardsjohn martin.github.io/MagPhyx/ (accessed June 12, 2019).

${ }^{3}$ D. Stauffer and A. Aharony, Introduction to Percolation Theory (CRC Press, 1994).

${ }^{4}$ B. F. Edwards, D. M. Riffe, J. Ji, and W. A. Booth, "Interactions between uniformly magnetized spheres,” Am. J. Phys. 85(2), 130-134 (2017).

${ }^{5}$ B. F. Edwards and J. M. Edwards, "Dynamical interactions between two uniformly magnetized spheres," Eur. J. Phys. 38(1), 015205 (2016).

${ }^{6}$ B. F. Edwards and J. M. Edwards, "Periodic nonlinear sliding modes for two uniformly magnetized spheres," Chaos 27(5), 053107 (2017).

${ }^{7} \mathrm{~S}$. Qu, "The Zen gallery" (2014), see http://zenmagnets.com/gallery/ (accessed May 20, 2019).

${ }^{8}$ B. F. Edwards, "Educational value of neodymium magnet spheres: Expert report in the matter of Zen Magnets, LLC, CPSC Docket No. 12-2, 10/20/2014 (Redacted)" (2014), see https://drive.google.com/file/d/OBw7DdocNZGQgWThT b3VvUHYza2s/view (accessed May 20, 2019).

${ }^{9} \mathrm{~J}$. Weis and D. Levesque, "Chain formation in low density dipolar hard spheres: A Monte Carlo study,” Phys. Rev. Lett. 71(17), 2729 (1993).
${ }^{10} \mathrm{~A}$. Clarke and G. Patey, "Ground state configurations of model molecular clusters," J. Chem. Phys. 100(3), 2213-2219 (1994).

${ }^{11}$ R. Messina, L. A. Khalil, and I. Stanković, "Self-assembly of magnetic balls: From chains to tubes," Phys. Rev. E 89(1), 011202 (2014).

${ }^{12}$ C. L. Hall, D. Vella, and A. Goriely, "The mechanics of a chain or ring of spherical magnets," SIAM J. Appl. Math. 73(6), 2029-2054 (2013).

${ }^{13}$ D. Vella, E. du Pontavice, C. L. Hall, and A. Goriely, "The magneto-elastica: From self-buckling to self-assembly," Proc. R. Soc. A 470(2162), 20130609 (2014).

${ }^{14} \mathrm{~N}$. Vandewalle and S. Dorbolo, "Magnetic ghosts and monopoles," New J. Phys. 16(1), 013050 (2014).

${ }^{15}$ J. Boisson, C. Rouby, J. Lee, and O. Doaré, "Dynamics of a chain of permanent magnets," Europhys. Lett. 109(3), 34002 (2015).

${ }^{16}$ J. Schönke and E. Fried, "Stability of vertical magnetic chains," Proc. R. Soc. A 473(2198), 20160703 (2017).

${ }^{17}$ G. L. Pollack and D. R. Stump, "Two magnets oscillating in each other's fields," Can. J. Phys. 75(5), 313-324 (1997).

${ }^{18}$ P. R. Bevington and D. K. Robinson, Data Reduction and Error Analysis for the Physical Sciences (McGraw-Hill, 2003). 